\title{
Die Quellen zum Kral-Spital in Konstantinopel*
}

\author{
Von U. B. Birchler-Argyros
}

Das Quellenmaterial zur byzantinischen Spitalgeschichte hat sich seit Beginn des 20. Jahrhunderts erfreulich vermehrt. Es erstaunt deshalb nicht, daß gerade in den letzten Jahrzehnten die Sekundärliteratur über dieses Thema beträchtlich zugenommen hat $(2 / 3 / 8 / 9 / 36 / 42 / 51)$.

Seit dem 7.Jahrhundert können in Ostrom gut organisierte Spitäler nachgewiesen werden, und seit dem 10. Jahrhundert fehlt es auch nicht an Hinweisen, die einen Unterricht an diesen Institutionen vermuten lassen. Mit Sicherheit kann ein solcher bisher nur im Pantokratorkrankenhaus des 12. Jahrhunderts und im Kral-Spital des 15. Jahrhunderts nachgewiesen werden. Etwas optimistisch folgert Miller aus diesen beiden Quellen, daß man alle größeren Spitäler auch als medizinische Ausbildungszentren zu betrachten habe:

"In sum, the whole tradition of Greek medicine and of hospital development points to the xenon as the place most suitable for physicians to teach both medical theory and practice. Although only those sources have survived which demonstrate that the twelfth-century Pantocrator Xenon and the fifteenth-century Krales Xenon maintained medicals schools, most probably all the large hospitals of Constantinople supported teaching programs." (36/ S. 158)

Es ist Aufgabe dieser Arbeit, den Quellen über diesen Kral nachzugehen, in der Hoffnung, dabei Genaueres über diese «Medical School» zu erfahren, aber auch eventuell zu hohe Vorstellungen abzubauen.

Das Kral-Spital wurde vom serbischen König oder Kral, Stefan Uros II., genannt Milutin (1282-1321), in Konstantinopel errichtet. Der Kral, das Wort soll übrigens von Karl, das heißt Karl dem Großen, abstammen, näherte sein mächtiges serbisches Reich kulturell stark an Byzanz an, und die Errichtung eines Spitals inmitten der Hauptstadt der Griechen mag der Absicht entsprungen sein, diese Macht und Größe der Serben selbst hinter den Mauern Konstantinopels deutlich aufzuzeigen.

Die erste Erwähnung des Xenons oder Krankenhauses finden wir 1321 in den Akten des Athosklosters Chilandar. Dieser Konvent wurde von Milutin

* Gewidmet meinem Lehrer, «phôs kai bathron kai patêr» Bertrand Bouvier, um die Worte des Apostolis zu gebrauchen. Besonders möchte ich Terpsichore danken, ohne deren Mithilfe diese Arbeit nicht entstanden wäre. 
stark gefördert, und wir können in der Klosterkirche eine zeitgenössische Abbildung des Herrschers betrachten (Abb.1) ${ }^{1}$. Kaiser Andronikos II. Palaiologos (1282-1328), der Verfasser der Urkunde, bestätigt die Besitzverhältnisse des Chilandarklosters und räumt dem Abt das Recht ein, bei seinen Aufenthalten in der Hauptstadt drei Zellen mit einer Kirche unter den Häusern im Hofe des Kral für sich zu beanspruchen ${ }^{2}$.

Es muß sich also um einen größeren Gebäudekomplex gehandelt haben. Noch im selben Jahr verfaßte Andronikos eine weitere Urkunde, worin der Kral erwähnt wird, welcher zusammen mit dem Chilandarkloster das Dorf Mamytzona in Mazedonien besaß ${ }^{3}$. Dieser gemeinsame Besitz scheint schnell zu Unstimmigkeiten geführt zu haben, denn schon im folgenden Jahr mußte er erneut definiert werden. Das Dokument von 1322 gibt uns folgende drei Informationen: Erstens besaß das Krankenhaus nicht nur zwei Drittel des Dorfes Mamytzona, sondern noch weitere Ländereien und Güter, vermutlich in der Nähe von Konstantinopel; zweitens verwaltet das Spital selbst seine Eigentümer, es war also eine juristische Person; drittens, und dies ist besonders interessant, es wird durch den Abt des Prodromos-inPetra-Klosters vertreten, wo die Unterhandlungen stattgefunden haben ${ }^{4}$.

Die letzte Chilandarakte, worin unser Spital erwähnt wird, entstand 1323. Ein Beamter ${ }^{5}$ wurde vom Kaiser beauftragt, ein genaues Inventar der Güter des Xenon und des Chilandarklosters in Mamytzona zu erstellen. In der Einleitung erfahren wir, daß er zwei Protokolle verfaßt hat, eines zuhanden des Xenon und das vorliegende für das Chilandarkloster. Zudem erwähnt er ein Chrysobull, das heißt eine kaiserliche Urkunde, vermutlich von Andronikos II., in welcher die Besitztümer des Krankenhauses bestätigt werden ${ }^{6}$. Wenn uns beide Urkunden auch verlorengegangen sind, so bezeugt uns ihre Existenz doch zumindest, daß sich auch die griechischen Kaiser schon zu Beginn des 14. Jahrhunderts um das Kralspital gekümmert haben.

Betreffs der Ländereien des Xenon können wir aus dem zitierten Dokument ableiten, daß das Dorf Mamytzona nur einen Teil seiner Güter repräsentiert. Dank der folgenden Aufzählung des Chilandaranteiles wissen wir, was dem Spital jährlich zustand, das ja genau das Doppelte erhalten sollte. Abgesehen davon ist das Dokument sozialgeschichtlich interessant, da es uns einen Einblick in die Familien und Vermögensverhältnisse eines griechischen Dorfes des 14. Jahrhunderts erlaubt. Die fünf Seiten umfassende Urkunde soll kurz zusammengefaßt werden (41, S.194-198). Das Chilandarkloster besaß 128 hörige Bauern (paroikoi); diese lebten in 36 Haushaltungen (selten mehr als zwei Kinder) und ingesamt 43 Häusern. Die 


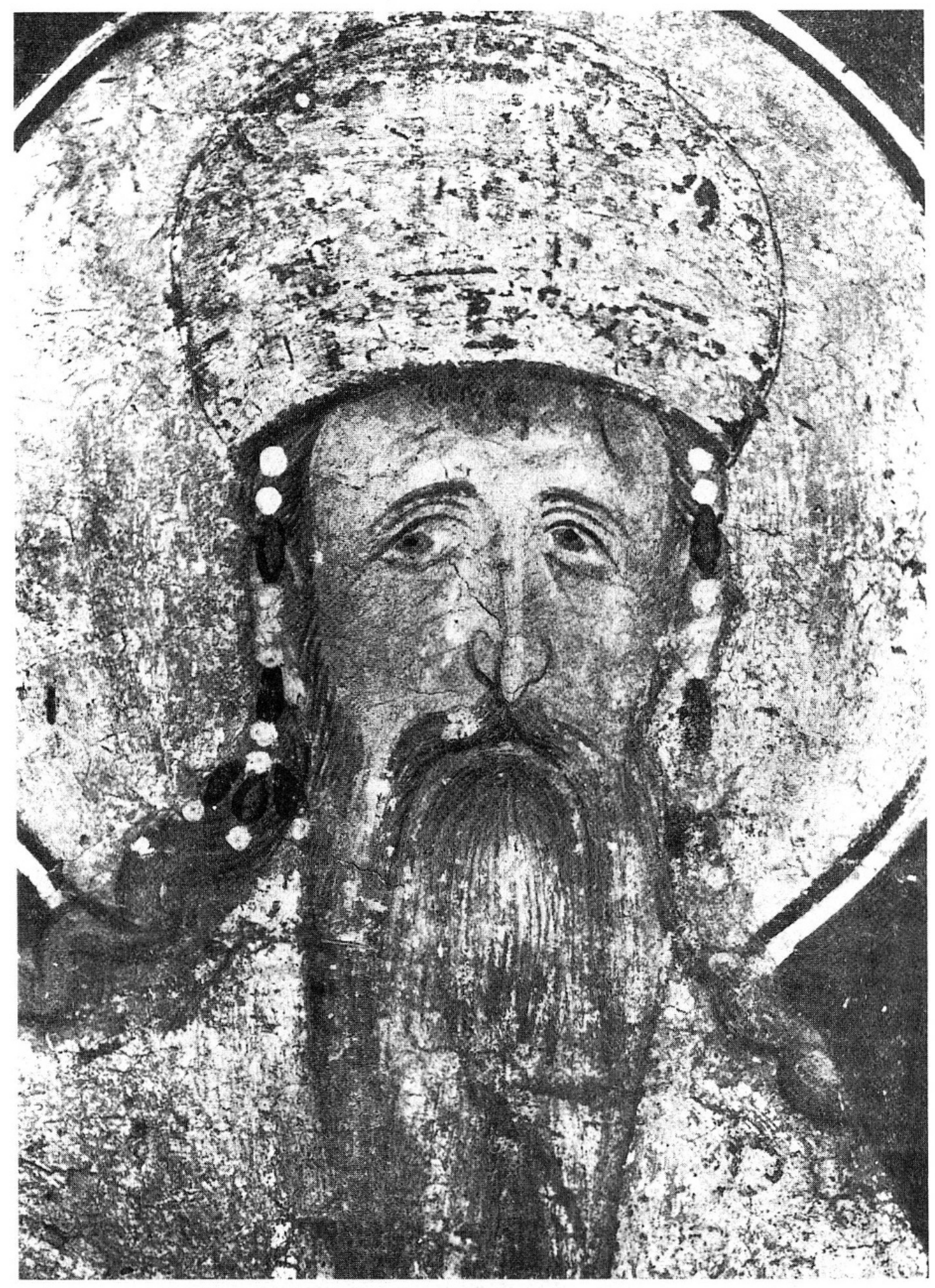

Abbildung 1: Der Kral (König) der Serben, Stafan Uros II., genannt Milutin (1282-1321). Zeitgenössisches Fresco des etwa siebzigjährigen Herrschers in der Klosterkirche (Katholikon) von Chilandar (15, Fig. 22) 
Grundstücke sowie andere Einnahmequellen wie Wind- und Wassermühlen der einzelnen Familien werden genauestens aufgezählt. Die jährlichen Steuereinnahmen aus dem einen Drittel des Dorfes betrugen 145 Goldmünzen.

Der Kralxenon, dem die restlichen zwei Drittel gehörten, bezog also jährlich rund 300 Hyperpyra von Mamytzona. Vom kleinen zeitgenössischen Lips-Krankenhaus (12 Betten) wissen wir, daß es über ein jährliches Einkommen von 600 Goldmünzen verfügte $(2, \mathrm{~V} / 3, \mathrm{~S} .71)$, doch dürfen wir nicht vergessen, daß das Dörflein in Mazedonien nur einen kleinen Teil des Gesamteinkommens darstellte.

Soviel in den Akten des Chilandarklosters. Die folgende Quelle ist für die Medizingeschichte die wichtigste. Es handelt sich um die rund zehn Jahre später, um 1332 geschriebene Biographie Milutins. Der Verfasser war dessen Zeitgenosse und hat den Herrscher persönlich gekannt. Es war der Erzbischof der serbischen Kirche, Danilo II. (um 1270-1337, Abb.2). Danilo schreibt:

«Und sogar in Konstantinopel selbst erbaute er an einer Stelle, genannt Prodromos ein Gotteshaus, indem er dafür unzählbares Geld stiftete; dort errichtete er auch viele prächtige Gebäude und gründete Xenodochien (ksenodohije), das heißt Hospitale (bolnica).

Hier stellte er eine überaus große Zahl von Krankenbetten auf und stattete sie mit weichen Matratzen aus. Und wenn ein Kranker keine Hoffnung mehr hegte, befahl er jedem, ein so vorbereitetes Krankenbett aufzusuchen.

Und von der griechischen Regierung erwarb er käuflich viele ausgesuchte Dörfer, vermachte sie (dieser Stiftung), damit sie dieser Institution Abgaben entrichten. Er suchte auch viele erfahrene Ärzte zusammen, gab ihnen viel Gold und was sie sonst benötigten, damit sie ständig die Kranken beaufsichtigen und sie heilen.

Und außerdem bestellte er für diese seine geeigneten und treuen Männer, welche die Kranken versorgen und für sie alles Nützliche verrichten, so daß sich kein einziger Kranker beklagen kann, da man ihm, wenn er um etwas bittet, auch gibt.

Dieser gütige Diener des christlichen Glaubens machte sich mit Lust die Worte der Schriften des Herrn zu eigen und war bestrebt, sie an sich selbst zu erfüllen, wie es im Evangelium heißt: Kommet, ihr Gesegneten meines Vaters, in das Reich, das seit Weltenschöpfung für euch bereitsteht; denn ich war hungrig, und ihr gabt mir zu essen; ich war durstig, und ihr gabt mir zu trinken; ich war krank, und ihr besuchtet mich.

Betrachtet, Geliebte, das Ausmaß der Güte dieses Christusliebenden und staunet, wie ungeheuer groß die Zahl der Kranken war, die an dieser Heilstätte untergebracht wurden; ist denn einer von ihnen übersehen worden, oder hat man ihn durch irgendeine Mißachtung gekränkt? Hatte einer von ihnen etwa etwas von seiner Bitte nicht erfüllt erhalten?

Im voraus ausgebildete und gütige Wohltäter dieser Frommen erschienen bei ihnen, und wenn einer dieser Kranken genas, ging er frohen Mutes seiner Wege; und es kam ein 


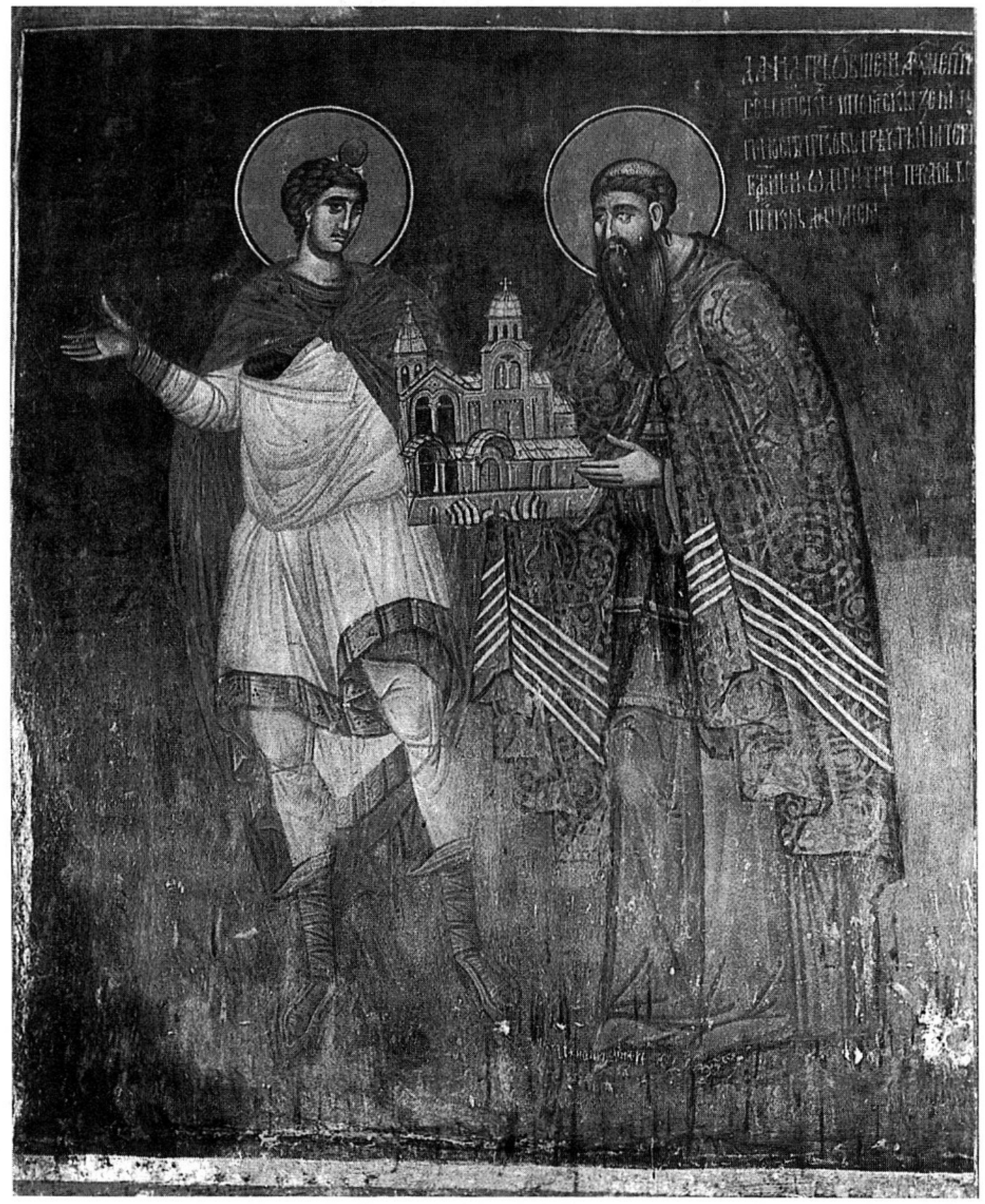

Abbildung 2: Stifterporträt des Erzbischofs Danilo II. (ca. 1270-1337) mit dem Propheten Daniel (li) aus dem 14. Jh. Westwand der Marienkirche des Patriarchats von Pec, wo sich auch sein Grabmal befindet (40, Abb. 13) 
anderer Kranker an seine Stelle und wurde genauso wohltätig versorgt, und so blieb es bis zum heutigen Tage unverändert zum erhabenen und wunderbaren Andenken und zum Lobe dieses frommen Königs (Stefan) Uros (II. Milutin).

An diesem Ort, beim Prodromoskloster werden, wie es dieser Fromme bei Lebzeiten bestimmte, bis zum heutigen Tag täglich viel Wein, Brot und andere verschiedene Lebensmittel zur Klosterpforte gebracht, damit es den Armen, Fremden und Bedürftigen reichlich verteilt wird, denn er (der König) wünschte sich im ewigen Leben immer wieder von neuem den Lohn Christi, des Herrn aller.» (21, S.177 ff.)

Vergleichen wir das Pantokratorstatut oder das des zeitgenössischen LipsSpitals mit diesem Text, wird man unschwer erkennen, daß wir es mit einem gut organisierten Krankenhaus der byzantinischen Spätzeit zu tun haben.

Die Bettenzahl wird leider nicht genau definiert und mit überaus gro $\beta$ angegeben. Viele erfahrene Ärzte und als Pfleger ausgebildete und gütige Wohltäter aus der Reihe seiner geeigneten und treuen Männer bildeten den Personalbestand. Es handelt sich also um geschultes Laienpersonal wie in den anderen Krankenhäusern, das entlöhnt wird. Aufnahme und Kost waren sicher unentgeltlich, wenn gesagt wird, daß sich kein einziger Kranker beklagen kann, da man ihm, wenn er um etwas bittet, auch gibt. Wie im Kosmosoteira-Spital (2, IV/3, S.65/51, S.200) wird ausdrücklich bemerkt, $\mathrm{da} \beta$ freigewordene Betten sofort neu besetzt werden sollen, es handelte sich somit um eine Art «Akutspital». Der Kralxenon war wie andere byzantinische Krankenhäuser eine königliche Stiftung, und eines der vielen ausgesuchten Dörfer, die Milutin der Institution vermachte, haben wir in Mamytzona kennengelernt. Ebenso wird hier bestätigt, daß es sich um einen größeren Gebäudekomplex gehandelt hat, wenn Danilo sagt, daß er viele prächtige Gebäude und ein Gotteshaus errichtete.

Die Chilandarakten haben uns gezeigt, daß der Kral administrativ dem Prodromos-in-Petra-Kloster unterstand. Danilos Angaben erlauben uns die genaue Lokalisation des Spitals. Es befand sich an einer Stelle genannt Prodromos, was wir zweifellos mit dem genannten Kloster identifizieren dürfen. Die jugoslawische Forscherin Mirjana Zivojinovic, die sich eingehend mit dem Kral befaßt hat, geht so weit, daß sie das oben genannte Gotteshaus mit der Prodromoskirche selbst identifiziert. Sie vermutet, daß Milutin das 1308 durch Feuer schwer beschädigte Kloster restaurierte, was ihm das Recht eingeräumt hätte, in dessen Nähe ein Krankenhaus zu gründen (52).

Da das Prodromoskloster im Zusammenhang mit dem Kral eine wichtige Rolle spielt, soll kurz dessen Geschichte erwähnt werden. Über seine Ur- 
sprünge wissen wir nichts Genaues, vermutlich gehen sie ins 6. Jahrhundert zurück. Berühmt wird die Anlage in spätbyzantinischer Zeit, wozu der dem Kloster benachbarte Blachernenpalast, der seit den Komnenen als kaiserliche Residenz diente, sicher einiges beigetragen hat. Vom Patriarchen Neilos (1379-1388) erhält der Prodromos 1381 den dritten Rang unter den Klöstern der Hauptstadt. Manuel II. Palaiologos (1391-1425) beschenkt es 1395 reichlich im Andenken an seinen serbischen Schwiegervater Konstantin Dragasès, was ein Hinweis auf eine serbische Tradition des Klosters ist (27, S.421).

Für unser Thema besonders wichtig war die Bibliothek des Klosters, von der noch heute 28 Manuskripte, verstreut in aller Welt, bekannt sind, darunter der Wiener Dioscurides. Er befand sich vermutlich seit der Wiedereroberung Konstantinopels nach dem lateinischen Kaiserreich (1261) im Besitz des Prodromos. Um 1360 wurde er von einem Mönch Neophytos ${ }^{9}$ kopiert (Cod. Paris. gr. 2286), welcher selbst ein botanisches Werk verfaßt hatte $(27$, S. $426 / 50$, S. 64 ff. $)$.

Als der Codex in Verfall geriet, erhielt Johannes Chortasmenos 1406 den Auftrag, das Buch zu restaurieren. Er hinterließ uns dabei auf Folio 1 recto des Codex Medicus Vindobonensis graecus No. 1 folgende Notiz:

Das vorliegende Buch des Dioscurides, das sehr alt ist und Gefahr lief, völlig zugrunde zu gehen, hat Johannes Chortasmenos gefestigt auf Kosten und Ermahnung des unter den Mönchen sehr geehrten Herrn Nathanael, zur Zeit Spitalverwalter (nosokomos) ${ }^{8}$ am Xenon des Kral. Im Jahre 6914, 14 Indiktion (1406). (14/30, Einl. S.24)

Die zitierte Stelle versichert uns, daß der Kral zu Beginn des 15. Jahrhunderts, also rund 100 Jahre nach seiner Gründung, noch in Betrieb war. Spitalverwalter war Nathanael ${ }^{9}$, ein Mönch des Prodromosklosters. Zudem deutet diese Quelle darauf hin, daß die Bibliothek des Prodromos auch vom Spitalpersonal konsultiert wurde. Chortasmenos (ca. 1370-ca. 1437) ist von der neueren Byzantinistik näher erforscht worden. Er war ein eifriger Handschriftensammler, Philosoph und Lehrer unter anderem des später berühmten Kardinals Bessarion. Johannes Chortasmenos verfaßte ein kleines Gedichtlein über einen Xenon, und es ist natürlich nicht unwahrscheinlich, daß es sich dabei um den Kral handelt ${ }^{10}$.

Einer Notiz, ähnlich derjenigen des Wiener Codex, begegnen wir noch einmal im Jahre 1442. Die Handschrift, die ebenfalls der Prodromos- bzw. Kral-Bibliothek angehörte, enthält Liturgisches und befindet sich heute in Ochrid. Auf Seite 25 des Pentecostarium Inv. 24 (alte Nummer 27) finden wir folgenden Satz: 
Dies schrieb Demetrios Angelos, Arzt am Xenon des Kral, mit Hilfe und auf Kosten des Spitalverwalters (nosokomos), des vornehmen (archontos) Herrn Laskaris. Der dies geschrieben hat, wird leider bald zu Staub und Erde eingeschlossen (egkleistheis?) im Grab. Diese Schrift aber wird ewig bleiben. Darum erinnert euch eher an den, der dies geschrieben hat, als an den Spitalverwalter, der dies bestellt hat, der allerdings auch bereitwillig den Lohn bezahlt hat:

Es wurde im Monat Januar der 10 Indiktion des Jahres 6950 (1442) beendet, in der Zeit, als die Muslime mit ungeheurer Kraft gegen Morea (Peloponnes) gezogen sind, um das Hexamilion zu zerstören, das von unserem glücklichen Gebieter und Herrn Konstantin Porphyrogennetos erbaut wurde. $\left(27\right.$, S.211) ${ }^{11}$

Es folgt nun der zweite Teil der Handschrift, der wiederum liturgischen Inhalts ist. Auf Seite 468 finden wir erneut eine Notiz des Schreibers:

Dieses Buch wurde durch die Hand des Demetrios Laskaris Angelos des Grafen (komitou) beendet, dank der Sorge und auf Kosten des Herrn Theodoros, des vornehmen (archontas) Laskaris, des Spitalverwalters; und durch unseren Fleiß.

Dies wurde am 1. des Monats Februar der 10 Indiktion geschrieben im Jahre 6950 (1442), als die Muslime das Hexamilion zerstörten, das gerade vom Herrn und Gebieter Konstantin dem Palaiologen fertig gebaut wurde ... das vierte ... ${ }^{12}$.

Darunter finden sich in roter Tinte geschrieben, stark verbleicht und schwer zu entziffern, zwei Zeilen, von denen man folgendes lesen kann:

«... gewidmet wurde das vorliegende Buch im ... des Kral.» (37, S.211)

Der Wiener Dioscurides und das Ochrider Pentecostarium zeigen, daß sich die Nosokomen des Kral in besonderem Maße um die Bibliothek gekümmert haben. Wie Nathanael war auch Theodor Laskaris ein Mönch (kyrios), dessen adelige Herkunft (archontas) betont wird.

Unser Schreiber, der «Arzt am Xenon des Kral» Demetrios Angelos Laskaris, hat nicht nur Handschriften kopiert, sondern besaß eine eigene medizinische Bibliothek. Er ist gewiß identisch mit jenem Demetrius Angelos, den wir als Eigentümer zweier medizinischer Codices kennen ${ }^{13}$.

Wir begegnen ihm gleich noch einmal in unserer letzten Quelle zum Kral. Diesmal handelt es sich um eine Miniatur ${ }^{14}$ im Codex Baroccianus 87 aus Oxford, der zwischen 1445 und 1453 entstanden sein muß (Abb. 3). Das Bild zeigt den Lehrer Johannes Argyropoulos, sitzend auf einem Thron, einer klassischen Stellung der byzantinischen Gelehrten-Ikonographie (43). In seiner Rechten hält er eine Art Szepter, mit der Linken weist er auf ein offenes Buch hin (homônuma legetai hôn onoma monon? Aristoteles Kategorien). Er trägt einen Kopfschmuck, der stark an denjenigen des Theodor Metochites auf einem Mosaik der Chorakirche erinnert. Zu Füßen 


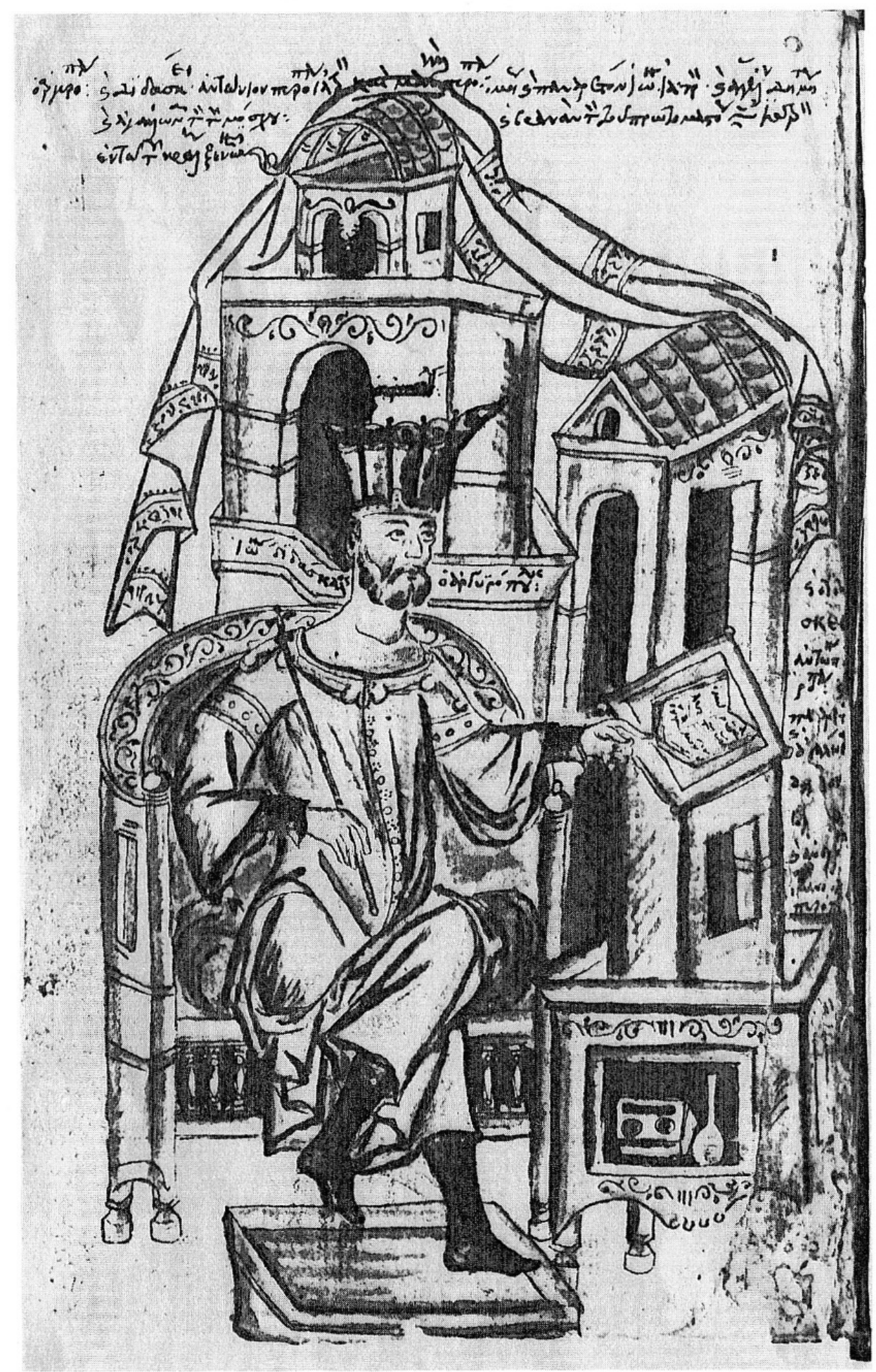

Abbildung 3: Johannes Argyropoulos, im Hintergrund die Gebäude des Kral. Miniatur des Oxforder Codex (Ms. Barocc. 87, fol. 33v). Originalgröße $200 \times 125$ mm, Foto der Bodleian Library mit freundlicher Druckerlaubnis 
des Lesepultes Schreibutensilien. Im Hintergrund die Gebäude des Kral, denn in der Überschrift heißt es:

Argyropoulos unterrichtet den Arzt Antonius Pyropoulos und Manuel Pyropoulos und den Arzt Johannes Panaretos und Angelos Demetrios und Agallon, Sohn des Moschos, und den Arzt Branas den Sohn des ersten Meisters (Prôtomastoros) im Xenon des Kral.

Hinter dem Kopf der Abbildung steht: «Der Lehrer (didaskalos) Johannes Argyropoulos» und am rechten Bildrand eine Wiederholung der Überschrift: «und er unterrichtet Antonios Pyropoulos und Panaretos und Agallon und Angelos und Branas und Andreas ${ }^{15}$ und Manuel Pyropoulos» (30, Einl. S.23).

Der Kral war also Sitz einer Schule. Der Verbindung Kloster-Bibliothek-Spital-Schule begegnen wir nicht zum erstenmal. Vermutlich schon im Magnanenkomplex (11.Jh.) vorhanden (18, S.25/27, S. 75 ff.), wird sie deutlich im Pantokrator (1136). Leider gibt uns das Pantokratorstatut keine Einzelheiten über den medizinischen Unterricht. Wir wissen nur, daß ein ausgezeichnet besoldeter Lehrer (didaskalos) damit betraut war, die jungen Ärzte (paidas tôn iatrôn) zu unterrichten (didaskein ta tês iatrikês epistêmês mathêmata). Ob dies nun ein rein theoretischer Unterricht im mittelalterlichen Sinne war oder ob ein praktischer Unterricht am Krankenbett stattfand, wissen wir nicht. Die Tatsache, daß die im Pantokratorspital arbeitenden Ärzte neben oder nach ihrer theoretischen Ausbildung einen Turnus in den verschiedenen Abteilungen ausführten, macht eine praktische Ausbildung sehr wahrscheinlich, beweist aber nicht einen «klinischen Unterricht», verstanden im heutigen Sinn (19, S. 106). Genauere Details erfahren wir über eine Schule in Konstantinopel um 1200, wo neben anderen Fächern auch Medizin gelehrt wurde. Nikolaos Mesarites (*ca. 1216/22) beschreibt lobend diese Institution, die sich in den Vorhallen der berühmten Apostelkirche befand ${ }^{16}$. Wiederum handelt es sich um eine Kirche mit einer Schule, wobei allerdings die Elemente Bibliothek und Spital fehlen. Dem von Mesarites beschriebenen Unterricht fehlt jeder Hinweis auf eine praktische Unterweisung der Ärzte (iatrôn paides). Wenn auch der Unterricht in gelösterem Rahmen und mit Diskussionen (syllogoi) erteilt wurde, bleibt er doch rein theoretisch (22, S. 90 ff.) Die Schule des Planoudes (ca. 1260-ca. 1310), aus der der bekannte Arzt Johannes Aktuarios hervorging, war ebenfalls einem Kloster angegliedert samt Bibliothek und vermutlich einem Xenon (18, S. $58 \mathrm{ff} . / 24$, S. 302). Deutlich wiederum liegt die Verbindung vor uns im Kral, wo wir alle Elemente - Kloster, Bibliothek, Spital und Schule nachweisen können. 
Was haben wir nun unter dieser Kral-Schule zu verstehen? Die einzigen Hinweise verdanken wir Johannes Argyropoulos (1393/4-1487) selbst und seinem Schüler Michael Apostolis (ca. 1422-1462). Geboren in Konstantinopel, wurde Argyropoulos von seinem Lehrer, vielleicht dem genannten Johannes Chortasmenos selbst, speziell gefördert. Neben seinen Studien begann er eine klerikale Laufbahn, wurde Priester und war auf dem Unionskonzil von Ferrara-Florenz (1438/39) anwesend. Nach 1444 eröffnete er eine Privatschule (mouseion) in Konstantinopel und wurde darauf vom Kaiser (Johannes VIII. Palaiologos 1425-1448) an eine Schule (didaskaleion) von Konstantinopel berufen, wo er, wie er selbst bezeugt, Philosophie unterrichtete $\left(7 / 30 / 32 / 33\right.$, S. 44/38) ${ }^{17}$. Michael Apostolis, ebenfalls Philosoph und nach dem Fall der Stadt als Handschriftenkopist in Kreta tätig, verdanken wir die aufschlußreiche Schrift: «Anrede an meinen Lehrer Johannes Argyropoulos, welcher auf Berufung des Kaisers im Katholikon Mouseion des Xenon unterrichtete.» ${ }^{18}$

Der Xenon, zweifellos identisch mit dem Kral, war also Sitz des «Katholikon Mouseion», einer vom Kaiser geförderten Schule. Verschiedene Byzantinisten glauben, im Kral die letzte Universität von Ostrom lokalisieren zu können (4, S.405/18, S.71/33, S.46/51, S. 195/52). Kaiser Manuel II. Palaiologos, den wir bereits als Gönner des Prodromosklosters kennengelernt haben, reorganisierte, beeindruckt von seiner Europareise, wo er unter anderem an der Sorbonne verweilte, das Bildungswesen in Konstantinopel. In der Folgezeit pilgerten viele Lateiner, insbesondere Italiener, für einen Bildungsaufenthalt nach Konstantinopel. So berichtet Francesco Filelfo (1398-1481) von seinen Studien an der «Universitas litterarum et scientiarum» (publicus discendi ludus). Der Byzantinist Louis Bréhier identifiziert diese Universität mit dem Kral und schreibt:

Au lieu d'écoles séparées, de caractère indéterminé, tous les enseignements furent réunis dans le même édifice... Le local choisi pour abriter l'Université était un hôpital fondé par le kral serbe Ourosch II Miloutine, adjoint au monastère de Saint-Jean-Baptiste. Ce choix, qui paraît étrange, peut s'expliquer par une raison d'économie, mais aussi par l'importance qu'avaient prise les études médicales. $(4$, S.405)

In der Folge zitiert er eine Reihe berühmter Persönlichkeiten, die am Kral unterrichtet haben sollen, wie Georgios Chrysococces, den schon genannten Chortasmenos, Georgios Scholarios (der spätere Patriarch Gennadios), Johannes Argyropoulos und Michael Apostolis. Unter den Schülern finden wir 
Männer wie Bessarion, Guarino di Verona, Francesco Filelfo und Konstantin Laskaris.

Sicher haben alle diese Persönlichkeiten in Konstantinopel doziert oder studiert, doch dürfen wir nicht vergessen, daß es dort noch eine ganze Reihe anderer Schulen gegeben hat $\left(18\right.$, S.63/45, S.34/ siehe auch $\left.{ }^{17}, 18\right)$. Es ist natürlich möglich, daß diese Personen am Kralxenon waren, als Sitz des Katholikon Mouseion aber kann dieser erst seit Argyropoulos mit Sicherheit nachgewiesen werden. Wurden nun alle Fächer an dieser Schule unterrichtet, und war der Kral eine Medical School, wie Bréhier und Miller behaupten?

Die einzigen Hinweise darüber finden wir wiederum bei Argyropoulos selbst.

Argyropoulos hatte vor seiner Berufung an den Xenon in Padua studiert, wo er uns 1443 als «Artistorum et Medicorum rector» begegnet (7, S. $24 \mathrm{ff} . / 30$, Einl. S.18 $)^{19}$. Nach dem Fall Konstantinopels wirkte er als Philosophieprofessor in Florenz und später am Hofe Sixtus IV. (1471-1484) in Rom, wo er als über 90 jähriger starb ${ }^{20}$. Der Titel «Medicorum rector» muß im zeitgemäßen Kontext verstanden werden. In Padua gab es zur Zeit des Argyropoulos zwei Studentengruppen, die Juristen und die Artisten, worunter auch die Mediziner gehörten. «Artistorum et Medicorum rector» heißt weder, daß Argyropoulos Rektor im heutigen Sinne war, noch daß er Arzt war, sondern einzig, daß er von den Studenten der Artistengruppe zu ihrem Führer gewählt wurde $(6$, S.338). In der Tat hat Argyropoulos während seiner Laufbahn ausschließlich aristotelische Philosophie unterrichtet, wobei, und das muß unterstrichen werden, die Medizin damals als Teil der Philosophie verstanden wurde.

Philosophie unterrichtete Argyropoulos, wie er selbst sagt, auch im $\mathrm{Kral}^{17}$, und der genannte Oxforder Codex enthält ja seine Vorlesungen über das Organon des Aristoteles. Daß ein Großteil der Hörer aus Ärzten zusammengesetzt war, zeigt uns die Miniatur. Antonios Pyropoulos, Johannes Panaretos und Branas werden eindeutig als solche bezeichnet. Daß auch Angelos Demetrios Arzt war und im Kralspital arbeitete, haben wir in der Ochrider Handschrift gesehen.

Ist nun die Tatsache, daß ein Teil des Auditoriums aus Ärzten zusammengesetzt war, ein Beweis, daß Medizin unterrichtet wurde? Sein Schüler Demetrios Angelos zum Beispiel mußte seine medizinische Ausbildung schon seit Jahren abgeschlossen haben, denn wir sind ihm ja bereits 1442 als Arzt im Kral begegnet. Waren die im Auditorium vertretenen Ärzte nicht eher an Philosophie interessierte Hörer als «Medizinstudenten»? 
Es ist sicher, daß Argyropoulos im Kral medizinische Probleme, im Rahmen der Philosophie doziert hat, denn wir finden in der vatikanischen Bibliothek einen Codex mit seiner Vorlesung über den Puls, aufgezeichnet von einem Schüler ${ }^{21}$. Zwei der in der Miniatur genannten Ärzte sind uns später als Verfasser medizinischer Werke bekannt (29/31/48/49) ${ }^{22}$; doch wird uns all dies nicht darüber hinwegtäuschen, daß der Unterricht ein philosophischer war, und von einem praktischen oder gar «klinischen Unterricht» der Medizin ist in den Quellen nirgends die Rede!

Bréhiers Ansicht, daß im Katholikon Mouseion ähnlich wie an der Apostelkirche alle Lehrfächer unter einem Dach vereint worden sein sollen, muß meiner Meinung nach Hypothese bleiben. Sicher bezeugt ist nur ein Ein-Mann-Unterricht in Philosophie vor einem mehrheitlich aus zum Teil bereits fertig ausgebildeten Ärzten zusammengesetzten Auditorium. Eine praktische Ausbildung im Spital, wie wir sie vom Pantokrator her kennen, ist anzunehmen. Beweise für eine Medical School oder einen praktischen Unterricht, wie ihn Miller als selbstverständlich voraussetzt (36, S. 158), fehlen aber bisher.

Mit dem Oxforder Kodex haben wir die Reihe der mit bekannten Quellen zum Xenon des Kral abgeschlossen. Wir wollen kurz die wichtigsten Informationen zusammenfassen:

- Das Kralkrankenhaus wurde vom serbischen König (Kral) Stefan Uros II., genannt Milutin, vermutlich zu Beginn des 14. Jahrhunderts gestiftet und mit den nötigen finanziellen Mitteln ausgestattet.

- Als funktionierendes Spital ist der Kral seit seiner Gründung bis zum Fall der Hauptstadt (1453), also rund 150 Jahre lang, nachzuweisen.

- Das Krankenhaus bestand aus einem ansehnlichen Gebäudekomplex (Hof, Kirche, mehrere Häuser) im Palastviertel von Konstantinopel. Es lag beim Kloster Prodromos-in-Petra, dem es administrativ unterstellt war.

- Es war ein «Akut-Spital», worin Kranke «in großer Zahl» aufgenommen wurden. Ärzte, die entlöhnt werden, und Pflegepersonal können nachgewiesen werden.

Namentlich bekannt sind die Spitalverwalter (nosokomoi) Nathanael (1406) und Theodor Laskaris (1442), beides Mönche, sowie der Arzt Demetrios Angelos Laskaris (1442). 
- Das Krankenhaus besaß «viele ausgesuchte Dörfer», die Milutin der griechischen Regierung abgekauft hatte. Wir wissen, daß ihm Ländereien in den Vororten (von Konstantinopel) gehörten und in der Nähe von Parapolia (Mazedonien). Vom Dorf Mamytzona, ebenfalls bei Parapolia gelegen, wissen wir, daß es dem Xenon jährlich 300 Goldmünzen (Hyperpyra) abzuliefern hatte.

- Ein Chrysobull, vermutlich von Andronikos II., das die Schenkungen Milutins bestätigt, muß bestanden haben, ebenso ein Inventar des Mamytzona-Besitzes. Beide sind verlorengegangen.

- Vor dem Fall der Hauptstadt (1453) beherbergte der Kral in seinen Mauern das «Katholikon Mouseion». Ob es sich dabei um eine Universität gehandelt hat, konnte ich nicht nachweisen. Sicher bezeugt ist ein philosophischer Unterricht vor einer mehrheitlich aus zum Teil fertig ausgebildeten Ärzten zusammengesetzten Hörerschaft. Ein praktischer medizinischer Unterricht im Krankenhaus kann zur Zeit ebensowenig bewiesen werden wie das Vorhandensein einer «Medical School».

- Mit Sicherheit kann nur ein Lehrer der Philosophie am Kral nachgewiesen werden: Johannes Argyropoulos (1393/4-1487).

- Folgende Schüler wurden am Kral von Argyropoulos unterrichtet:

- Antonius Pyropoulos, Arzt und Verfasser medizinischer Werke ${ }^{22}$.

- Demetrios Angelos, Arzt am Krankenhaus des Kral und Besitzer medizinischer Handschriften ${ }^{13}$.

- Johannes Panaretos, Arzt und Schreiber philosophischer Werke ${ }^{22}$.

- Branas, Arzt und Sohn des Protomastoras(?), vermutlicher Autor medizinischer Werke ${ }^{22}$.

- Manuel Pyropoulos

- Andreas Pyropoulos

- Agallon, Sohn des Moschos

Als Schüler des Argyropoulos in Konstantinopel sind der Philosoph Konstantin Laskaris (1434-1501) und Gian Mario Filelfo (1426-1480), der Sohn des Francesco (1398-1481), und Michael Apostolis (ca. 1422-1462) bekannt. Allerdings wissen wir nicht, ob diese im Kral oder in einem der vielen anderen Mouseia unterrichtet wurden ${ }^{17}$.

- Das Krankenhaus oder das Kloster besa $\beta$ eine Bibliothek, die von beiden vermutlich gemeinsam benutzt wurde, wobei die Spitalverwalter als «Bibliothekare» eine wichtige Rolle spielten. 
Nach Einnahme der Stadt durch die Türken wurde das Prodromoskloster durch die Janitscharen geplündert und schnell dem Verfall anheimgestellt (27, S. 424/44, S. 152). Letztmals wird es von Stefan Gerlach 1578 erwähnt, also rund 120 Jahre später, als es bereits in Ruinen lag (10, S. 190).

Es ist möglich, daß die «Waagschalenmoschee» oder Kefeli Camii, nicht unweit der berühmten Pammakaristoskirche gelegen, zum Prodromos-inPetra-Kloster gehört hat. Der Bau, ein langgestrecktes Gebäude mit zwei Fensterreihen und einem Holzdach, der zwischen dem 9. und 12. Jahrhundert datiert wird und nur eine Apsis an der Nordseite besitzt, diente vielleicht als Refektorium, wobei man auch an den Xenon selbst denken dürfte ${ }^{23}$. Dies ist allerdings eine reine Hypothese, denn eine exakte Lokalisation ist den Archäologen bis heute nicht gelungen (16, S.14/17, S.321/27, S.427).

\section{Anmerkungen}

(Die Übersetzung der griechischen Texte stammt vom Autor)

1 Milutin errichtete zahlreiche Kirchen und Klöster sowohl in Serbien als auch in Thessaloniki, Konstantinopel und Jerusalem. Sechs Kirchen sind uns erhalten geblieben: Die Klosterkirche in Chilandar, die Kirche der Gottesmutter von Ljeviska in Prizren, die Klosterkirche des hl. Nikita bei Skopje, die Königskirche im Kloster Studenica, die Georgskirche in Staro Nagoricane und die Klosterkirche in Gracanica. Bis auf die Kirche des hl. Nikita finden wir in allen diesen Gebäuden ein Bildnis des Königs (28).

2 Urkunde No.58 Anno 6829, Monat Februar, 4 Indiktion (1321): Andronikos II. Palaiologos bestätigt die Besitztümer von Chilandar in Kasandria, Kalamaria, Thessaloniki und am Strymon und bewilligt dem Abt des Chilandarklosters die Nutznießung von drei Zellen im Spital, welches vom König der Serben in Konstantinopel gegründet wurde.

... Zudem wurde verordnet, daß der jeweilige Abt des genannten ehrwürdigen Chilandarklosters drei Zellen zu seiner Verfügung haben soll, in welchen sich auch eine Kirche befindet. Diese Zellen sind in den Häusern (hospêtia), welche sich im Hof des Krankenhauses (xenôn) befinden. Jenes Xenons, welcher vom serbischen Kral, meinem Sohn und kaiserlichen Schwiegersohn (Milutin heiratete die fünfjährige Simonis, die Tochter des Andronikos II. Palaiologos) in der glorreichen, von Gott geschützten und groß gemachten Stadt Konstantinopel errichtet wurde.

In diesen Zellen soll sich der Abt ausruhen und aufhalten, wenn er sich einer Arbeit wegen in dieser Stadt aufhält. Ich der Kaiser befehle mit diesem Chrysobull, daß es nur dem Abt von Chilandar erlaubt sein soll, in diesen Zellen zu verweilen, und daß niemand anders, sei es von Serbien oder von meinen Untertanen, das Recht haben soll, darin zu wohnen ... (41, S. 138 und 139) 
3 Urkunde No.60 Anno 6829, Monat Juni, 4 Indiktion (1321): Auf Anfrage des Königs der Serben bestätigt Andronikos II. Palaiologos dem Chilandarkloster seine Besitztümer von Kalamaria am Strymon und an anderen Orten.

... Dem Chilandarkloster gehört zudem die Hälfte des Dorfes Mamitziona ${ }^{7}$, welches sich in der Gegend von Parapolia (Stadt in Mazedonien) befindet, mitsamt seinen Rechten und Weidegründen. Die andere Hälfte des Dorfes gehört in derselben Art dem Xenon, welcher vom serbischen Kral, meinem Sohn und kaiserlichen Schwiegersohn, in der glorreichen, von Gott geschützten und groß gemachten Stadt Konstantinopel errichtet wurde. Denn dieser hat, wie schon gesagt wurde, durch vorherigen Kauf all diese Beitztümer dem Kloster vermacht zu seinem Seelenheil ... (41, S. 142)

Dieses Chrysobull wird wortwörtlich und mit demselben Datum von Andronikos III. (1328-1341) bestätigt (41, S.144). Wegen seines schlechten Lebenswandels versuchte Andronikos II. seinen Nachfolger zuerst vom Throne auszuschließen. Mit Hilfe Milutins aber hat sich Andronkos III. behaupten können und wurde 1324 zum Mitkaiser gekrönt.

4 Das für die Kralgeschichte wichtige Dokument sei hier ausführlich wiedergegeben:

Urkunde No.82 1 August, 5 Indiktion (1322): Abkommen zwischen den Chilandarmönchen und den Verwaltern des serbischen Spitals von Konstantinopel betreffs der Teilung des Dorfes Mamytzona:

+ Gemäß dem Inhalt des göttlichen und verehrten Chrysobulls, das uns von unserem mächtigen heiligen Herrn und Kaiser (Andronikos II.) ausgestellt wurde, werden dem trefflichen und wohltätigen Spital Grundstücke (ktêmeta) in den Vororten und Pachtgüter zuerkannt und gewidmet. Dieses Spital wurde vom großen Kral von Serbien, dem fromm dahingeschiedenen Sohn und Schwiegersohn unseres mächtigen Herrn und Kaisers (Andronikos II.) errichtet. (Milutin war in der Zwischenzeit gestorben 1321)

$\mathrm{Zu}$ den genannten Grundstücken und Vororten wurden noch zwei Drittel des Dorfes Mamytzona hinzugefügt ${ }^{7}$. Das eine Drittel wurde dem ehrwürdigen Kloster der allerheiligsten Gottesmutter und Beschützerin, Chilandar, gewidmet. Wir sind erneut am 1 August der 5 Indiktion (1322) zusammengesessen, um dieselbe Frage gemeinsam zu prüfen:

- Ich Meletios, Mönch, Vorsteher des ehrwürdigen und kaiserlichen Klosters, das den Namen des heiligen und glorreichen Propheten Johannes trägt, des Vorläufers und Täufers, genannt in Petra

- zusammen mit dem würdigen unter den Mönchen, Herrn Kallinikos (kyr Kallinikos) ${ }^{6}$

- und zusammen mit dem Abt des ehrwürdigen Klosters der allerheiligsten Gottesmutter und Beschützerin, Chilandar, Herrn Gerbasios

- und zusammen mit diesem war auch Herr Theophilos, der würdige unter den Mönchen

- in dieser Absicht sind auch die wichtigsten und ehrwürdigsten Männer des Prodromos zusammengekommen.

Gemeinsam haben wir bestätigt und beschlossen, was schon vorhin gesagt wurde, gemäß dem Inhalt des göttlichen und verehrten Chrysobulls. Das gesamte Dorf Mamytzona soll in jeglicher Beziehung und Art vom wohltätigen Spital verwaltet 
werden: Damit meinen wir jegliche Arbeit, jegliche Fürsorge, wie Aussaat, Ausgaben oder irgendwelche andere Arbeit, die notwendig ist.

In der Erntezeit kommen dem wohltätigen Spital zwei Drittel der Früchte und der Getreide zu, die er in seine Kasse (tamieion) tun soll. Das andere Drittel soll bedingungslos und unverzüglich dort abgegeben werden, wo es der Abt des Chilandarklosters verteilen will. Dies ist so.

Sollte sich später mit Gottes Willen das Dorf Mamytzona vergrößern, sei es durch Ankauf von Reben, Obstgärten oder Gemüsegärten, und wenn es sich auch nur um ein Zehntausendstel vergrößern oder verbessern sollte im Vergleich zum jetzigen Zustand, so müssen dennoch diese Bedingungen und Worte unverbrüchlich eingehalten werden, das heißt: das wohlfährtige Spital soll zwei Teile davon erhalten und den dritten das Chilandarkloster.

Die Verwaltung (hupêresia) und Administrierung (hupourgia) des Dorfes aber soll, wie schon beschlossen, ganz in den Händen des Spitales liegen. Da also durch gemeinsamen Entschluß und vollständiges gegenseitiges Einverständnis diese Bedingungen und Worte als gut und tadellos bestätigt wurden, soll es von jetzt an und in Ewigkeit beiderseits und unabänderlich und unverbrüchlich eingehalten werden ...

Zur Bestätigung und Versicherung des oben genannten wurde dieser Brief geschrieben und dem wohltätigen Kloster der allerheiligsten Gottesmutter, genannt Chilandar, gegeben zur Verhinderung jeder Belästigung.

Meletios, Abt und Mönch +

Gerbasios, Ekklesiarchis und Mönch

+ Markianos, Skevophylax und Levitis

+ Gennadios, Mönch

+ Mitrophanis, Mönch (41, S.175-177)

5 Leider fehlt in der Quellenausgabe von Louis Petit die Unterschrift, die im Original vorhanden sein muß (41, S. 194 Anm.).

6 Urkunde No.92 Anno 6832, Monat September. 7 Indiktion (1323): Ein kaiserlicher Beamter überträgt Kallinikos einen Drittel des Dorfes Mamitzona.

+ Ich habe den göttlichen und verehrungswürdigen Auftrag erhalten, der vorschreibt, sämtliche bei Parapolia liegenden Grundstücke des Xenon zu überprüfen und zu gliedern, des wohltätigen Xenon, welcher in der von Gott geschützten, glorreichen und von Gott regierten Stadt Konstantinopel errichtet wurde von jenem höchsten serbischen Kral, des Herrn Stephan Ouros, des Sohnes und kaiserlichen Schwiegersohnes unseres kräftigen heiligen Herrn und Kaisers. Ich werde all das, was diesem Xenon durch das göttliche, ehrwürdige und verehrungswürdige Chrysobull zugeschrieben wurde, mit Hilfe eines Protokolls (dia praktikou) aufzählen.

Von diesen Grundstücken soll ich das sogenannte Mamitzona ${ }^{7}$ in drei Teile einteilen. Zwei von diesem muß ich mit den übrigen Grundstücken dem genannten wohltätigen Xenon zuschreiben und übergeben.

Mit dem restlichen Drittel werde ich dasselbe tun durch ein anderes Protokoll und es dem Mönch Herrn Kallinikos (Mönch von Chilandar, siehe ${ }^{4}$ ) übergeben. Gemäß dem Inhalt des göttlichen und verehrungswürdigen Befehles habe ich das Dorf Mamitzona überprüft und gegliedert wie die anderen (Grundstücke). Von der 
Gesamtmenge des genannten Dorfes habe ich ihm (Kallinikos) einen Drittel gegeben, der folgendes enthält: ... (es folgt nun das detaillierte Inventar des dem Kloster zugeteilten Dorfdrittels) (41, S. 194)

7 Es existieren drei Schreibweisen in den Texten: Mamitziôna, Mamytzôna und Mamitzôna.

8 Nosokomos, ursprünglich der mit der Administration des Infirmariums betraute Mönch, wird in der Literatur oft mit Krankenwärter oder Arzt übersetzt. Miller (36, S. 115 und 162) glaubt, daß die Funktion des Nosokomen im Laufe der Zeit durch einen Laienarzt übernommen wurde. Meines Wissens ist dies ungewiß. Der Nosokome am Pantokrator hatte rein administrative Aufgaben, und auch die beiden Nosokomen des Kral waren Mönche.

9 Eine Urkunde des Prodromosklosters aus dem Jahre 1395 trägt die Unterschriften der Mönche Neophytos und Nathanael. Sie sind vermutlich identisch mit den zwei oben genannten Personen des Prodromos-Klosters (35, S.263).

10 Das Gedichtlein lautet:

Jambische Trimeter, geschrieben im Xenon, als die dort sich befindenden Arbeiter (banausoi) uns herauszuwerfen versuchten.

«Es ist tatsächlich ein fürchterliches und wahrhaft bitteres Leiden, wenn der Mann, der sich nach Weisheit sehnt, mit Arbeitern

und das Wort Hassenden zusammen ist.

Denn, wenn er den Bösen nicht schnellstens entgehen kann,

riskiert er vieles von ihnen zu erleiden.

Dies sage ich, auf mich anspielend.» (25, S. 195)

Ein weiteres Gedicht ist an einen Isidor des Spitals (tou nosokomeiou) gerichtet (25, S.196)

11 Kaiser Manuel II. Palaiologos (1391-1425) errichtete am Isthmus von Korinth eine Mauer, das Hexamilion. 1423 wurde dies erstmals von den Türken zerstört. Der spätere Kaiser Konstantin XI. Palaiologos (Porphyrogennetos) hat sie wieder aufgerichtet. Endgültig wurde das Hexamilion am 10. Dezember 1446 von Murad II erstürmt und zerstört (5, S. 392, 402, 406).

12 Das Datum ist zweifellos 1442. Es entzieht sich meiner Kenntnis, ob das Hexamilion unter Konstantin mehr als einmal zerstört wurde.

13 Der Besitzer der folgenden Bücher medizinischen Inhalts ist vermutlich mit unserem Demetrios Angelos identisch:

- Codex Parisinus graecus 2154 aus dem 14. Jh. mit Werken von Galen (de usu partium), Michael Psellos (Syntagma e libris medicis), Melampous (Opus de palpitationibus), eines anonymen Schriftstellers (de alimentis) und mit folgender Notiz:

«und ich, der Arzt Demetrios Angelos, habe dieses nebst anderen Büchern ihm abgekauft.» (13, S.X)

- Codex ambrosianus graecus 659, früher Q.3. Sup. aus dem 14. Jh. der Galens Kommentar zum Prorrheticum des Hippokrates enthält mit folgender Notiz:

Dieses Buch des Galen habe ich, der Arzt Demetrios Angelos, gekauft von dem Priester Herrn Papa Demetrios, Sohn des Medias, für 25 ottomanische Weißpfennige im Monat 
März des Jahres 6974 der 13 Indiktion (1466), als der große Herr (Mohamed II. der Eroberer 1451-81) gegen die Albaner auszog und bei seiner Rückkehr große Beute und viele Sklaven mitbrachte. $(12, \mathrm{~S} .31)$

14. Das Bild wurde erstmals von Hodius veröffentlicht (23, S. 195), dann von Legrand (32, S. 166 a) und S. Lampros (30, Bild 2).

15 Neu ist hier Andreas, der in der Überschrift nicht genannt wurde.

16 Wie die Hagia Sophia war die Apostelkirche ein Wahrzeichen der Hauptstadt. Von Konstantin dem Großen errichtet, wurde sie von Justinian von Grund auf erneuert. Architekt war Anthemios von Tralles, Bruder des berühmten Arztes. Konstantin, der «dreizehnte Apostel», war darin von 12 Scheinsärgen umgeben bestattet. Bis ins 9. Jahrhundert diente die Kirche als Grabstätte der Kaiser (die Komnenen ließen sich im Pantokrator-, die Palaiologen im Lips-Kloster beerdigen, beides übrigens Klöster mit Spitälern). Nach der Eroberung wurde die Kirche abgebrochen und durch die SüleymanMoschee ersetzt (1462). Der St. Markus-Dom in Venedig wurde nach dem Vorbild der Apostelkirche errichtet (22).

17 Ein Auszug des Briefes sei hier wiedergegeben, da er zur Biographie des Argyropoulos wichtig ist:

«Brief des weisen und gelehrten Herrn Johannes Argyropoulos, Diakon und Edler der Kirchen, an Georg von Trapezunt»

... Seit meiner Kindheit habe ich lange Zeit mit dem Studium an den Schulen (phrontistêria) verbracht. Ich habe dort Lehrer gehört, die sehr berühmt waren für ihre Weisheit. Dazu hatte ich eine sorglose und gute Natur, habe hinreichend Unterricht genommen und viel Fleiß aufgebracht. Ich war in dauernder Gesellschaft von Aristoteles und Plato.

Als ich meinen Lehrern gleichkam, wurde ich vom Kaiser, der auch in dieser Hinsicht sehr weise war, gewürdigt, der Schule (didaskaleion) vorzustehen. Viele Weise aus Italien, die uns zugehört haben, haben uns gelobt und unsere Kenntnis bewundert, insbesondere diejenige, die wir zeigten über die Physik und die Logik (syllogismoi) des Aristoteles ... (30, S. 73 und 74)

18 Wie die «Anrede an meinen Lehrer» bezeugt, war Michael Apostolis, genannt Byzantios (ca. 1422-1462), Schüler des Agyropoulos, den er als Leuchte, Fundament und Vater betitelt. Aus dem Text geht deutlich hervor, daß es mehrere Mouseia gegeben hat und daß Argyropoulos nach seiner Rückkehr aus Italien zuerst in einer eigenen Schule und danach im «Katholikon Mouseion» Philosophie doziert hat; von einem medizinischen Unterricht ist nirgends die Rede:

Als er aus Europa, wohin die griechische Kultur ausgewandert war, heimkehrte und den Zustand sah, in welchem sich die Mouseia befanden, die völlig vernachlässigt waren und nur noch den Namen von einem Mouseion bewahrt hatten, da empfand er Mitleid mit seinem geliebten Vaterland, dem es so schlecht ging, und er beklagte es, daß die Wissenschaften so verachtet wurden. Sogleich faßte der Edle den Entschluß, die Wissenschaften wiederherzustellen, und er eröffnete ein Mouseion, nicht wie er es gerne gehabt hätte, noch wie er es wollte, als er von Italien zurückkehrte, denn die Zeit schüttelte das wie das andere durcheinander. 
Dennoch ragte sein Mouseion weit über das hinaus, was man im Durchschnitt bei uns findet. Es war wie eine neue Akademie, Stoa oder Peripatos, eine Wohnstätte der Fürsten der Philosophie. Jene, die in Mengen dorthin strömten, hörten dort nicht dasselbe wie die anderswo Studierenden, sondern das, was von Homer, Demosthenes und Plato gesagt wurde.

Unser edler und die Philosophie liebender Kaiser hat dies gesehen und von geachteten Leuten gehört. Er sah ein, daß es nicht richtig war, daß diese Dinge auf dem Felde oder im Garten unterrichtet wurden, sondern sie müssen so unterrichtet werden, daß sie dem Vaterland Ehre bereiten, das heißt dort, wo ihr ihn jetzt seht, sitzend auf einem hohen Lehrstuhl (eph hypsêlou kathêmenon bêmatos), von wo aus er jene widerlegen kann, die von sich eine große Meinung haben, während sie nur weniges wissen und selbst dieses nicht richtig. Nicht strotzende gemeine Rethorik fließt von seinen Lippen, noch die Sprache der Dichter oder der Schriftsteller, sondern das, was wir als Dialektik bezeichnen, welche die Schönheit des Wahren ehrt und das Falsche zurückweist. Welcher vernünftige Mensch würde da nicht die Hände klatschen und möchte diese Seligkeit nicht besitzen, die von dort herabströmt dank der Weisheit des Lehrers und dank dem kaiserlichen Entschluß.

Wer vom kaiserlichen Hof, wer von der Straße, möchte dies nicht? Selbst die Handwerker und Arbeiter (banausoi), wenn sie Gefühl für das Schöne haben, werden durchdrungen vom Genuß der Schönheit ... (30, S. 228 ff. Dieser Text, von Apostolis selbst handgeschrieben, findet sich im Codex palatinus graecus 275 der vatikanischen Bibliothek 47, S.150).

Vor dem Fall der Stadt spielte Apostolis zusammen mit Argyropoulos eine Rolle als Anführer der lateinerfreundlichen Partei. Dies bezeugt ein Gedicht von Umbertino Pusculo aus Brescia, einem Zeitgenossen, der in Konstantinopel wohnte. Die Griechen waren in zwei politische Gruppen gespalten, jene die für die Union mit Rom plädierten, darunter Argyropoulos und Apostolis, und die Gegner, die in der Mehrzahl waren:

Johannes Argyropoulos, der Liebling der Musen und ausgezeichnet in der Kunst der Athene (Palladis arte), hat die meisten unterrichtet. Mit seinen Worten hat er in jener stürmischen Zeit versucht, die Gläubigen zu überzeugen, Gott und dem Papst treu zu bleiben. Ihm folgte (hunc sequitur) der würdige und hochgelehrte Michael Byzantinus mit dem Beinamen Apostolus (7, S.42).

Verschiedene Autoren betrachten Apostolis als Nachfolger des Argyropoulos im Kral (4, S.407/30, Einl. S. 14/33, S.46/38, S.144/49), indem sie das «hune sequitur» in diesem Sinne interpretieren, obwohl dies im Kontext politisch verstanden werden muß. Wenn Argyropoulos bis zum Fall der Stadt in Konstantinopel anwesend war (7, S.44/33, S.46), warum sollte dann Apostolis seine Nachfolge angetreten haben?

Sicher hat auch Apostolis in Konstantinopel unterrichtet, ohne jedoch topographische Angaben zu machen. Dies bezeugt sein Brief an die Lateiner, worin er diesen vorschlägt, in Italien eine Schule zu eröffnen:

Die Schüler (paides) sollen ungefähr 15jährig oder etwas jünger sein. Dazu müssen sie die Sprache der Römer beherrschen, so wie es jedem möglich ist. Sie müssen jene Bücher besitzen, die ich ihnen vorschreibe. Den ganzen Tag müssen sie mit mir 
verbringen. Am Mittag sollen sie etwas Leichtes essen, wie es üblich ist, danach müssen sie wieder zurück in die Schule (mouseion).

Ich werde nicht auf einem hohen Lehrstuhl sitzen (eph hypsêlou bêmatos) und schreien, sondern auf einem Sitz oder Schemel (thôkon ê tripoda) und auf gleicher Ebene mit ihnen.

Genauso habe ich es in Byzanz (Konstantinopel) und in Kreta gemacht. Deshalb hatte ich auch viele griechische und römische Studenten (phoitêtai), obwohl ich noch jung war und weniger wußte als die Älteren, die viel wußten $(38, \mathrm{~S} .153)$.

Apostolis unterrichtete wohl in einer Privatschule und war vermutlich noch zu jung, um vom Kaiser an den Kral berufen zu werden. Wie er im genannten Brief ausführt, lehrte er die Grundfächer mit Grammatik, Poesie, Rhetorik, Logik und Physik. Von einem medizinischen Unterricht finden wir auch hier keine Spur (38, S. 153).

19 In der «Antiaula magna» der Universität Padua finden wir eine sicher viel später entstandene Abbildung des Argyropoulos.

20 Domenico Ghirlandajo (1449-1494) hat Argyropoulos zweimal portraitiert.

- In der sixtinischen Kapelle auf seinem Fresco «La vocatione degli Apostoli Pietro e Andrea»: Argyropoulos befindet sich rechts neben den knienden Aposteln als zweiter in der Reihe der stehenden Männer mit weißem Bart (46, S. 55 Bild 1).

- Im Palazzo Vecchio in Florenz, Sala di Cosimo: Cosimo ist umgeben von berühmten Persönlichkeiten. Über seiner linken Schulter steht Argyropoulos wiederum mit Bart (7, Abb.).

21 Im Codex vaticanus graecus 285 I finden wir auf folio 151 recto folgende Einleitung:

Im Puls werden drei Ursachen als Hauptsächlichste betrachtet ... wie uns der Lehrer der Lehrer (ho tôn didaskalôn didaskalos) und wahrhaftig weiseste und gelehrteste Herr Johannes Argyropoulos ausdrücklich gelehrt hat. (34, S.396)

Ein gutes Beispiel dieser Philosophie-Medizin ist die vermutlich zur Kralzeit abgefaßte Abhandlung des Argyropoulos mit dem Titel: «Lösung auf die Fragen und Aufgaben, welche einer der Ärzte und Philosophen von Zypern stellte.» Die Fragen dieses Arztes sind vorwiegend theologisch-philosophischer Natur und enthalten nur am Rande medizinische Probleme (7, S.33/30, S. 140).

22 Verschiedene Quellen sprechen von einem Antonios Pyropoulos; ob es sich um dieselbe Person handelt, ist nicht sicher (29/31/48):

- Codex Vindobonensis medicus graecus 17: Geschrieben von einem Archiatros Pyropoulos. Er enthält 10 Bücher der Medizin des Pyropoulos (neos archiatros) (26).

- Codex Parisinus graecus No. 335 du Fond Coislin: Er enthält das Rezept eines Antonio Pyropoulos gegen den Husten (11, S.318). Lampros vermutet darin einen Auszug aus einem Jatrosophion, das sich im 16. Jh. im Besitz eines Michael Kantakouzinos befand: «Jatrosophion des weisen Pyropoulos. Das Buch ist groß und enthält viele durch die Erfahrung (ex empeirias) gemachte Tatsachen» (31, S.132).

- Ermolao Barbaro der Jüngere schreibt einen Brief an einen Antonios Pyropoulos (vor 1494), über den er schreibt: 
«Schon oft und viele haben wir gehört, die von deiner edlen Neigung zur Wissenschaft sprachen, dies in bezug auf das Allgemeinwissen wie auch der Mathematik und der Physik und derem wichtigsten Teil, der Medizin.» (31, S. 128)

- Eine weitere Notiz finden wir in einem Moskauer Kodex:

«dieses Buch der Auscultationes Physicae des Aristoteles gehört mir, dem kinderlosen nichtswürdigen und sündhaften Antonios Pyropoulos. Dies Buch wurde vom heiligen und weisen Herrn Patriarchen Gennadios von eigener Hand geschrieben. Ich widme es dem verehrungswürdigen heiligen kaiserlichen Kloster von Vatopedi (Athos) den 12 Oktober 7001, 12 Indiktion (1494)» (31, S.128)

Einem Johannes Panaretos (30, Einl. S.26/46) begegnen wir als Schreiber des Laurentianus 1 Pluteus 81 der Werke des Aristoteles enthält, der aber laut Bandini aus dem 12. oder 13. Jh. stammen soll $(1$, S.219).

- Der Codex Urbinatis graecus 123 der Bibliotheca Apostolica Vaticana war im Besitz eines Johannes Panaretos (20, S.447).

- Im Codex Vaticanus graecus 1679 des 12. Jh. finden wir auf Folio 2 und 349 Gebete eines Johannes Panaretos, dessen Schrift dem 14. Jh. zuzuordnen sei (20, S.447).

Von einem Branas ist uns ein Hustenrezept überliefert (48, S.232).

23 Das Kosmosôteira-Spital in Aenos war im Grundriß vermutlich ein langgestrecktes Rechteck mit einer eigenen Kapelle (2, IV/3, S.69).

\section{Literaturverzeichnis}

(1) Bandini A. M.: Catalogus Codicum Graecorum Bibliothecae Laurentianae T. 3, Florenz 1770.

(2) Birchler-Argyros U.B.: Quellen zur Spitalgeschichte im byzantinischen Reich 1980/81 (unveröffentlicht).

(3) Birchler-Argyros U.B.: Byzantinische Spitalgeschichte, ein Überblick. In: Historia hospitalium. Zeitschrift der deutschen Gesellschaft für Krankenhausgeschichte. Heft $15,1983 / 84$.

(4) Bréhier L.: La civilisation byzantine, Edition: L'évolution de l'humanité, A. Michel Paris 1950/70.

(5) Bréhier L.: Vie et Mort de Byzance, Edition: Lévolution de l'humanité, A. Michel Paris $1946 / 69$.

(6) Bylebyl J. J.: The School of Padua. In: Health, medecine and mortality in the sixteenth century. Edited by Ch. Webster. Cambridge University Press 1979.

(7) Cammelli G.: Giovanni Argiropulo. I dotti bizantini e le origini dell'umanismo II. Firenze 1941.

(8) Codellas P.: The Pantocrator the imperial byzantine medical center of 12 th century A.D. in Constantinople. In: Bulletin of the history of medicine Bd. 12, S.392-410 1942.

(9) Constantelos D. J.: Byzantine philantrophy and social Welfare. Rutgens University Press 1968.

(10) Crusius M.: Turcograecia, Basel 1584. 
(11) Devreesse R.: Catalogue des manuscrits grecs II. Le Fond Coislin. Paris 1945.

(12) Diels H.: Die handschriftliche Überlieferung des Galenischen Kommentars zum Prorrheticum des Hippocrates. In: Abh. der Kgl. Pr. Ak. der Wiss. 1912.

(13) Diels H.: Corpus Medicorum Graecorum Y.9.2. Praefatio: Galeni in Hippocratis Prorrheticum commentaria S.X. 1915.

(14) Dioscurides: Codex Yindobonensis Medicus graecus 1. Vollständige farbige Faksimile Ausgabe. Akademische Druck- und Verlagsanstalt Graz 1965/7.

(15) Djuric J. Y.: Fresques médievales à Chilandar. In: Actes du XII congrès international d'études Byzantines, Band 3, Ochrid 1961.

(16) Eyice S.: Istanbuler Moscheen, die ehemals Kirchen waren. In: TAC Têrkiye Anit Cevre Cilt: 1 Sayi: 2 Mai 1986 (türkisch).

(17) Freely J. et Sumner-Boyd H.: Istanbul, ein Führer. Übersetzung und deutsche Bearbeitung: Wolf-Dieter Bach. Prestal Verlag München 1975.

(18) Fuchs F.: Die höheren Schulen von Konstantinopel im Mittelalter. Byzantinisches Archiv (Zusatz zur Byzantinischen Zeitschrift Nr. 8), 1926.

(19) Gautier P.: Le typicon du Christ Sauveur Pantocrator. In: Revue des études byzantines Bd. 32, S. 1-145, 1974.

(20) Giannelli C.: Codices Vaticani Graeci (No. 1458-1683), Roma 1950.

(21) Hafner S.: Serbisches Mittelalter. Altserbische Herrscherbiographien. Bd.2. Danilo II. und sein Schüler: Die Königsbiographien. Übersetzt, eingeleitet und erklärt von Stanislaus Hafner. Verlag Styria, Graz/Wien/Köln, 1976.

In der Reihe: Slavische Geschichtsschreiber, herausgegeben von Günther Stökl, Bd. IX.

(22) Heisenberg A.: Grabeskirche und Apostelkirche, Leipzig Bd. II, 1908.

(23) Hodius: De Graecis Illustribus, London 1742.

(24) Hohlweg A.: Johannes Aktuarios, Leben - Bildung und Ausbildung. De Methodo Medendi. In: Byzantinische Zeitschrift, Bd. 76, 1983.

(25) Hunger H.: Johannes Chortasmenos. Wiener byzantinische Studien, Bd. 7, bei H. Böhlaus Nachf. Wien/Köln/Graz 1969.

(26) Hunger H.: Katalog der griechischen Handschriften der österreichischen NationalBibliothek. Codices jur. et med. Teil 2, Wien 1969.

(27) Janin R.: La géographie ecclésiastique de l'empire byzantin. Première partie: Le siège de Constantinople et le patriarchat cecuménique, Tome 3 , Les églises et les monastères 2 éd. Paris 1969.

(28) Jugoslawien: Ein enzyklopädischer Reiseführer. Yugoslaviapublic, Beograd 1983.

(29) Kouzês A.: Some new information on Anton Pyropoulos as physician according the Codex 877 of Iberian Monastery and Cod. med. gr. 27 of the national library of Vienna. Praktika Akad. Athen 194.6.

(30) Lampros S.: Argyropoulos, Hrsg. P. D. Sakellarios Athen 1910 (gr).

(31) Lampros S.: Antonios Pyropoulos. In: Neos Hellênomnêmôn, Bd. 10, 1913 (gr).

(32) Legrand M.: Bibliographie hellénique. $\mathrm{XV}^{\mathrm{e}}$ et $\mathrm{XV}^{\mathrm{e}}$ siécle Bd.3.

(33) Manoussakas M. et Staikos C.: L'attività editoriale dei Greci durante il Rinascimento Italiano (Ausstellungskatalog: «Firenze Capitale Europea della Cultura»), Atene 1986.

(34) Mercati J.: Codices Vaticani Graeci. Tomus I (No. 1-329), Rom 1923.

(35) Miklosich et Müller, Acata et diplomata, Bd.2.

(36) Miller Th.S.: The Birth of the Hospital in the Byzantine Empire. Bulletin of History of 
Medicine. New Series No.10. The Johns Hopkins University Press, Baltimore/London 1985.

(37) Mosin V.: Les manuscrits du Musée National d'Ochrida. Recueil de travaux. Bibliothèque A. Mirambel, Ochrid 1961.

(38) Noiret H.: Michael Apostolis, Lettres, Paris 1889.

(39) Ostrogorsky G.: Geschichte des byzantinischen Staates. Beck'sche Verlagsbuchhandlung München 1940.

(40) Petkovic S.: Das Patriarchat von Pec, Beograd 1982.

(41) Petit L.: Actes de Chilandar, Première partie: Actes grecs 1911.

(42) Philippsborn A.: Der Fortschritt in der Entwicklung des byzantinischen Krankenhauswesens. In: Byzantinische Zeitschrift, Bd. 54, S. 338-385, 1961.

(43) Pournaropoulos K.G.: Zwei Miniaturen byzantinischer Ärzte aus einem Kodex des 14. Jahrhunderts (Mitteilungen der griech. Gesellsch. für Gesch. der Med.), Athen 1977 (gr.).

(44) Runciman S.: Die Eroberung von Konstantinopel 1453. Übersetzt von Peter de Mendelssohn. Verlag C. H. Beck. München 1966.

(45) Speck P.: Die kaiserliche Universität von Konstantinopel. Byzantinisches Archiv (Zusatz zur byzantinischen Zeitschrift Nr. 14), C. H. Beck München 1974.

(46) Steinmann: Die Sixtinische Kapelle, München 1901.

(47) Stevenson H. sen.: Codices Manuscripti Palatini Graeci Bibliothecae Vat. Rom 1885.

(48) Trapp E.: Die Stellung der Ärzte in der Gesellschaft der Palaiologenzeit. In: Byzantinoslavica Bd.33/2, 1972.

(49) Trapp E.: Prosopographisches Lexikon der Palaiologenzeit, Wien 1978.

(50) Volk O.: Die byzantinischen Klosterbibliotheken von Konstantinopel, Thessalonike und Kleinasien (Dissertation an der Ludwig-Maximilians-Universität) München 1954.

(51) Volk R.: Gesundheitswesen und Wohltätigkeit im Spiegel der byzantinischen Klostertypika. Miscellanea Byzantina Monacensia Bd.28, München 1983.

(52) Zivojinovic M.: L'hôpital du roi Milutin à Constantinople (en serb.). In: Zbornik rad. Viz. inst. 16, S. 105-117 (mit französischer Zusammenfassung) 1975. 


\section{Summary}

Kral Hospital was an important institution in Constantinople before its fall under the Turks. It was founded under the Serbian King Stefan Uros II. Milutin (1282-1321) and was in working order for about 150 years, until the fall of the city. It used to be a stately building near the Blachernai, the Emperor's residence; its administration had presumably been put under the control of the monastery of "Prodromos in Petra", where the employees of Kral Hospital visited and used the library. "A large number of sick people", doctors and employees can be authenticated. We know by name two administrators (nosokomoi), Nathanael (1406) and the nobleman Theodoros Laskaris (1442), and a physician, Demetrios Angelos Laskaris (1442).

The hospital had its own landed territory and its properties in the Macedonian village Mamytzona are exactly known. Before the fall of the city, there used to be a school within the hospital where we can assert that philosophy, which includes also theoretical medicine, was taught by Johannes Argyropoulos (1393/4-1487). There is however no evidence for practical teaching or a "Medical School".

Dr. med. et med.dent. Urs Benno Birchler-Argyros

Kieferchirurgie FMH

2 , rue de la Pépinière

CH-1201 Genève 